\title{
Simulación de un actuador hidráulico sumergible para rehabilitación de rodilla
}

Simulation of a submersible hydraulic actuator for knee rehabilitation

Jhon Vera Luzuriaga. ${ }^{1}$, Jorge Sebastian Buñay Guaman. ${ }^{2} \&$ Edwin Rodolfo Pozo Safla. ${ }^{3}$

Recibido: 1-10-2019 / Revisado: 25-10-209 /Aceptado: 16-11-2019/ Publicado: 05-12-2019

Abstract.

DOI: https://doi.org/10.33262/cienciadigital.v3i4.2.1091

This article is a study through research simulation Design and Mechanical Simulation of a Submersible Rotary Hydraulic Actuator For Application in Hydrotherapy. This device that will help the rehabilitation of a knee is characterized by the necessary position and speed parameters according to the type of material used and that has the ability to be used in hydrotherapies. In the first stage of design two versions of the research project were taken to validate them, in the second stage it must be validated by software simulations according to the CAD model determined in the first stage. It is concluded that the validation of the model must be simulated subject to certain modified parameters to validate according to the rehabilitation.

Keywords: Rotary actuator, Simulation, Design, Hydraulic mechanics, Simscape, Multibody.

\section{Resumen.}

El presente artículo es un estudio por medio de simulación de la investigación Diseño y Simulación Mecánica de un Actuador Hidráulico Rotativo Sumergible Para Aplicación en Hidroterapia. Este dispositivo que va ayudar a la rehabilitación de una

1 Escuela Superior Politécnica de Chimborazo, Facultad de Mecánica. Riobamba, Ecuador. jhon.vera@espoch.edu.ec

${ }^{2}$ Escuela Superior Politécnica de Chimborazo, Facultad de Facultad de Mecánica. Riobamba, Ecuador.jorge.bunay@espoch.edu.ec

3 Escuela Superior Politécnica de Chimborazo, Facultad de Facultad de Mecánica. Riobamba, Ecuador. edwin.pozo@espoch.edu.ec 
rodilla se caracteriza los parámetros de posición y velocidad necesarios según el tipo de material empleado y que tenga la capacidad de ser usado en las hidroterapias. En la primera etapa de diseño se tomaron dos versiones del proyecto de investigación para validarlos, en la segunda etapa se debe validar por simulaciones por software según el modelo CAD determinado en la primera etapa. Se concluye que la validación del modelo debe ser simulado sometido a ciertos parámetros modificados para validar según sea la rehabilitación.

Palabras claves: Actuador rotatorio, Simulación, Diseño, Mecánica hidráulica, Simscape, Multicuerpo.

\section{Introducción.}

La dinámica multicuerpo nos permite desarrollar sistema mecánicos complejos, detallados para su análisis. En los últimos años el problema dinámico de sistemas complejos ha tenido gran impacto en los diseños de mecanismos y manufactura de productos de alta calidad. La simulación mediante software es una herramienta que todo ingeniero debe aplicar para obtener resultados aproximados a los esperados en la implementación real. Al momento de simular un producto es necesario conocer cada componente del producto. Los sistemas mecánicos multicuerpo referencia a los eslabones que relaciona a los componentes para que el programa de computadora pueda formular métodos de resolución y formulación de la generación de ecuaciones del movimiento cinemáticos y dinámicos. [1]

En el análisis de la mecánica multicuerpo es la comprensión del movimiento de los subsistemas (cuerpos o componentes). El movimiento de los materiales (cuerpos) formó el tema de algunas de las primeras investigaciones realizadas en tres campos diferentes: la mecánica de cuerpos rígidos, la mecánica estructural y la mecánica de continuidad. El término cuerpo rígido implica que la deformación del cuerpo en cuestión se supone pequeña, de modo que la deformación del cuerpo no tiene efecto en el movimiento corporal grueso. Por lo tanto, para un cuerpo rígido, la distancia entre cualquiera de sus dos partículas permanece constante en todo momento y en todas las configuraciones. El movimiento de un cuerpo rígido en el espacio se puede describir completamente utilizando seis coordenadas generalizadas.[2]

Parte de la investigación en dinámica de multicuerpos está dedicada a la selección de las coordenadas del sistema y los grados de libertad del sistema que se pueden usar de manera eficiente para describir la configuración del sistema. Se debe hacer una compensación entre la generalidad y la eficiencia de la formulación dinámica. Los métodos utilizados en el análisis dinámico de sistemas de cuerpos rígidos múltiples pueden, en general, dividirse en dos enfoques principales: El primero, la configuración del sistema se identifica utilizando un conjunto de coordenadas cartesianas que describen las ubicaciones y orientaciones de los 
cuerpos en el sistema. Este enfoque tiene la ventaja de que la formulación dinámica de las ecuaciones que gobiernan el movimiento del sistema es sencilla, en la segunda, las coordenadas relativas o conjuntas se utilizan para formular un número mínimo de ecuaciones dinámicas que se expresan en términos de los grados de libertad del sistema. En muchas aplicaciones, este enfoque conduce a una formulación recursiva compleja basada en ecuaciones de lazo cerrado. [2][3]

La rehabilitación postoperatoria para la artroplastia total de rodilla ha ganado importancia. Las demandas de disminución de la duración de la estancia hospitalaria y la reducción de los costos hospitalarios se han convertido en de suma importancia. La rehabilitación postoperatoria para la artroplastia total primaria de rodilla continúa estudiándose, por lo que el costo se reduce al mismo tiempo que proporciona la calidad de los resultados clínicos esperados por el cirujano y el paciente.

Un investigador introdujo el concepto de una técnica de caída y suspensión para el rango de movimiento (RDM) postoperatoria. Con esta técnica, la rodilla se cierra en flexión y el paciente flexiona activamente la rodilla a partir del primer día del postoperatorio hasta $90^{\circ}$ (comunicación verbal, K Bramlett, MD. Sugerido en el Simposio de la Fundación de Investigación de Cirugía de Implante de Articulación en Montreal, Canadá, agosto 1993). Funcionaría más rápidamente el uso de una máquina de movimiento pasivo continuo, lo que disminuiría la estadía y el costo en el hospital. Además, la RDM y la fuerza muscular se compararían a los 3 y 6 meses para determinar si se mejora la función final. [4]

El actuador hidráulico diseñado por el grupo de investigación después de pasar por dos prototipos de diseño se opta por el más compacto para el funcionamiento hidráulico según estudios de torque que puede generar el paciente bajo el agua, en hidroterapias.

En estudios que han realizado en el Instituto de Física y Escuela de Educación Física y Deporte, Universidad de Sao Paulo, São Paulo, Brasil dice que caminar en el agua se usa comúnmente como un ejercicio de bajo impacto para entrenamiento y rehabilitación (Heyneman y Premo, 1992; Prins y Cutner, 1999). Desde un punto de vista biomecánico, hay dos razones principales por las que caminar en el agua puede ser beneficioso: la disminución del peso corporal aparente debido a la fuerza de flotación y la mayor resistencia al movimiento debido a la fuerza de arrastre ejercida por el agua en el cuerpo humano. De hecho, el peso corporal aparente en el agua (la fuerza gravitatoria menos la fuerza de flotación) se reduce a aproximadamente un tercio del peso corporal cuando los sujetos caminan en agua de pecho y en la mitad del agua hasta la cintura. [5]

\section{Metodologia.}

El modelamiento matemático del servoactuador se lo realizara al trabajar sobre los subsistemas Mecánico e hidráulico. 
Modelamiento del subsistema mecánico.

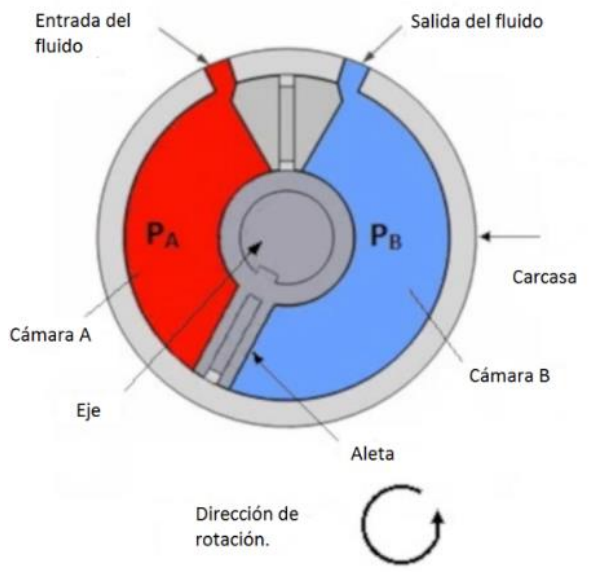

Fig. 1. División de la carcasa del actuador.

Fuente: Elaboración propia.

El Vane (Aleta) divide en dos cámaras la carcasa del actuador. El sistema tiene una entrada y una salida, el VANE será el elemento encargado de convertir la presión del fluido en energia mecánica de rotación en el eje. El torque $\tau$ a ser aplicado para el actuador rotatorio puede ser expresado por una fuerza media equivalente, a una fuerza $\mathrm{F}$ multiplicada por un a distancia media $\mathrm{r}_{\mathrm{m}}$, la cual es perpendicular a la línea de acción de la fuerza.

$$
\tau=\mathrm{Fr}_{\mathrm{m}}
$$

La Fuerza se expresa a través de la diferencia de presiones entre las cámaras del actuador hidráulico, el cual genera el movimiento rotacional sobre el eje. Para lo cual se considera la distribución de presiones sobre el VANE de forma uniformemente distribuida a lo largo de toda la superficie. Para el cálculo de la fuerza media, la superficie del Vane tiene una geometría aproximadamente rectangular y queda definida con los parámetros $R_{i}$ y $R_{e}$ y h.

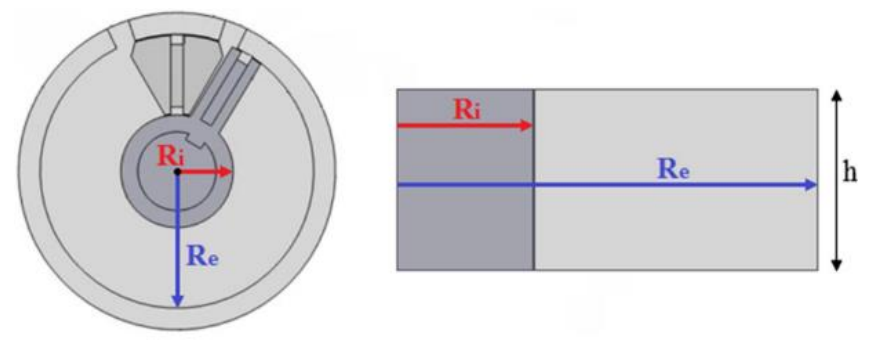

Fig. 2. Distribución de las fuerzas en el actuador.

Fuente: Elaboración propia.

Dónde:

$\mathrm{R}_{\mathrm{i}}$ Es el radio del eje

$\mathrm{R}_{\mathrm{e}}$ Es el radio desde el punto central del eje al extremo del Vane (Aleta) 
Y h es la altura de la Aleta.

El área de aplicación dela presión estará definida por:

$$
\mathrm{A}_{\mathrm{a}}=(\mathrm{Re}-\mathrm{Ri}) * \mathrm{~h}
$$

En tanto que el volumen $\mathrm{V}$ de las cámaras se calculara en función de la posición del Vane (Aleta) y variará de forma proporcional al desplazamiento del Vane. El área de la sección circular que forma cada una de las cámaras se determinara por la ecuación: $\mathrm{Sc}_{\mathrm{i}}=\theta *$ $\left(\mathrm{Re}^{2}-\mathrm{Ri}^{2}\right) / 2$ donde $\mathrm{Sc}_{\mathrm{i}}$ será la sección circular de cada cámara y $\theta$ es el complemento del arco que define la cámara.

Por lo tanto al multiplicar por la altura de la aleta se tendrá el volumen de la cámara.

$$
\mathrm{V}=\theta *\left(\mathrm{Re}^{2}-\mathrm{Ri}^{2}\right) * \mathrm{~h} / 2
$$

La fuerza $\mathrm{F}$ estará determinada por la diferencia de presiones $\Delta_{\mathrm{p}}$ entre las cámaras multiplicada sobre el área afectiva del Vane.

$$
F=A_{a} * \Delta_{p}
$$

Por lo tanto el torque estará dado por:

$$
\tau=\left(R e^{2}-R i^{2}\right) * \frac{h}{2} * \Delta_{p}
$$

Dinámica rotacional del actuador:

$$
\tau=J \frac{d^{2} \theta}{d t^{2}}
$$

Dónde:

$J$ : Es el momento de inercia de la parte móvil del actuador con relación al eje sobre el cual gira el actuador.

Al remplazar el valor de $\tau$ sobre la ecuación se tendrá:

$$
0.5 *\left(R e^{2}-R i^{2}\right) * h * \Delta_{p}=J \frac{d^{2} \theta}{d t^{2}}
$$

Dinámica de la presión en las cámaras del actuador:

Consideraciones para el estudio hidrodinámico:

El fluido es incompresible.

La distribuciones de presiones en el Vane es uniforme.

El modelo no tiene filtraciones. 


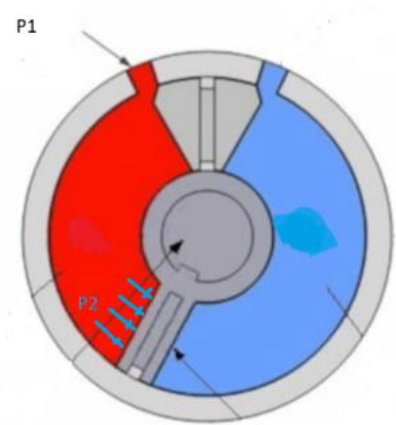

Fig. 3. Distribución de las presiones en el actuador.

Fuente: Elaboración propia.

Al aplicar el teorema de Bernoulli en los dos puntos del cilindro.

$$
\begin{gathered}
\frac{V_{1}^{2} \rho}{2}+P_{1}+\rho g z_{1}=\frac{V_{2}^{2} \rho}{2}+P_{2}+\rho g z_{2} \\
P_{1}-P_{2}=\frac{V_{2}^{2} \rho}{2}-\frac{V_{1}^{2} \rho}{2}+\rho g z_{2}-\rho g z_{1} \\
\Delta_{p}=\frac{V_{2}^{2} \rho}{2}-\frac{V_{1}^{2} \rho}{2}+\rho g z_{2}-\rho g z_{1}
\end{gathered}
$$

Dónde:

$$
V_{2}=R * \omega
$$

Esta velocidad será función de la ubicación del punto de análisis así como también el valor de $\mathrm{z}_{2}$.

$$
\Delta_{\mathrm{p}}=\frac{\rho\left(\mathrm{V}_{2}^{2}-\mathrm{V}_{1}^{2}\right)}{2}+\rho g\left(\mathrm{z}_{2}-\mathrm{z}_{1}\right)
$$

Si ponemos el sistema de referencia en el punto $\mathrm{z}_{1}$ por lo tanto $\mathrm{z}_{1}=0$ con lo cual:

$$
\Delta_{\mathrm{p}}=\frac{\rho\left(\mathrm{V}_{2}^{2}-\mathrm{V}_{1}^{2}\right)}{2}+\rho \mathrm{gz}_{2}
$$

El valor de $\mathrm{V}_{1}$ dependería de los parámetros de la unidad de potencia y la válvula solenoide que se utilice para el control.

Al considerar $\omega=\frac{d \theta}{d t} y V_{2}=R * \omega$ sobre la ecuación:

$$
0.5 *\left(R e^{2}-R i^{2}\right) * h * \Delta_{p}=J \frac{d^{2} \theta}{d t^{2}}
$$


Se tendría.:

$$
0.5 *\left(R e^{2}-R i^{2}\right) * h *\left(\frac{\rho\left(R^{2} * \frac{d \theta}{d t}-\mathrm{V}_{1}^{2}\right)}{2}+\rho \mathrm{gz}_{2}\right)=J \frac{d^{2} \theta}{d t^{2}}
$$

La velocidad de rotación dependerá del caudal en la entrada V1.

\section{Modelado de la fricción generada en el Van del actuador servo-hidráulico}

El fenómeno físico de la fricción se presenta en elementos mecánicos que se encuentran en contacto y cuando existe entre los mismos un movimiento relativo entre los mismos. De acuerdo a Beater, El modelamiento matemático de la fricción es complejo debido a que se trata de un fenómeno aleatorio y variante en el tiempo.

Además la fricción tiene características que dependen de ciertos factores como la velocidad relativa de los cuerpos, la temperatura, la dirección de desplazamiento, la lubricación y las condiciones de rugosidad de la superficie.

En los actuadores hidráulicos la fricción se presenta principalmente entre el contacto entre el Oring ubicado en el Vane y la cámara del actuador.

Para el presente estudio se asume la hipótesis en la que la fricción es similar a la de un actuador lineal hidráulico. El modelo de fricción usado es el de LuGre propuesto por Canudas de Wit et al, las cuales serán modificadas para ser aplicadas a un modelo rotacional.

El modelo LuGre presume que las superficies en contacto presentan rugosidades, donde el contacto entre las irregularidades impide el deslizamiento entre las mismas.

El modelo se enfoca en el caso en el que los cuerpos están inicialmente en reposo hasta que una acción externa ocurre, con lo cual tienden a existir un movimiento relativo entre ellos. Esto lleva a una deformación elástica entre los elementos en contacto, donde se produce una región que genera una fuerza que obstruye el movimiento entre los dos cuerpos en contacto.

En el modelo clásico, la ecuación matemática de LuGre, la fricción, además de un término de fricción, es compuesta de una fuerza que es proporcional a una medición indirecta del micro-deformación relacionado a los elementos en contacto. Esta fuerza se compone por tres partes: la primera es calculada directamente a través de las micro-deformaciones, la otra es relacionada a su primera derivada con respecto al tiempo, y la última es asociada con la de fricción debida a la viscosidad. En este estudio las ecuaciones son usada en forma rotacional por lo que se usa un Torque por fricción en el modelo por lo cual se usa la ecuación siguiente:

$$
T_{f r i}=\sigma_{0} z+\sigma_{1} \frac{d z}{d t}+\sigma_{2} w
$$


Donde $T_{f r i}$ es el torque debido a la fricción en el actuador, $\sigma_{0}$ representa el coeficiente de rigidez de la deformaciones microscópicas entre la superficie de contacto del actuador, y $z$ es la media de las micro-deformaciones de los picos relacionados al mecanismo del actuador. Adicionalmente $\sigma_{1}$ es el coeficiente de amortiguamiento, $\sigma_{2}$ representa el amortiguamiento de la viscosidad, y $w$ representa la velocidad relativa del actuador.

La dinámica de la media de las micro-deformaciones $z$ está definida por las Eq:

$$
\frac{d z}{d t}=w-\frac{|w| \sigma_{0}}{g(w)} z .
$$

El termino $g(w)$ el representa el efecto Stribeck, es positivo y depende básicamente de las propiedades del material, lubricación y temperatura. La ecuacion muestra la parametrización del término $g(w)$.

$$
g(w)=T_{c}+\left(T_{s}-T_{c}\right) e^{-\left(\frac{\omega}{\omega_{s}}\right)^{2}}
$$

Al combinar las ecuaciones se obtendrá la ecuación:

$$
T_{s S}=\operatorname{sgn}(\omega)\left(T_{c}+\left(T_{s}-T_{c}\right) e^{-\left(\frac{\omega}{\omega_{s}}\right)^{2}}\right)+\sigma_{2} w
$$

La cual representa el torque generado por la fricción en un estado permanente.

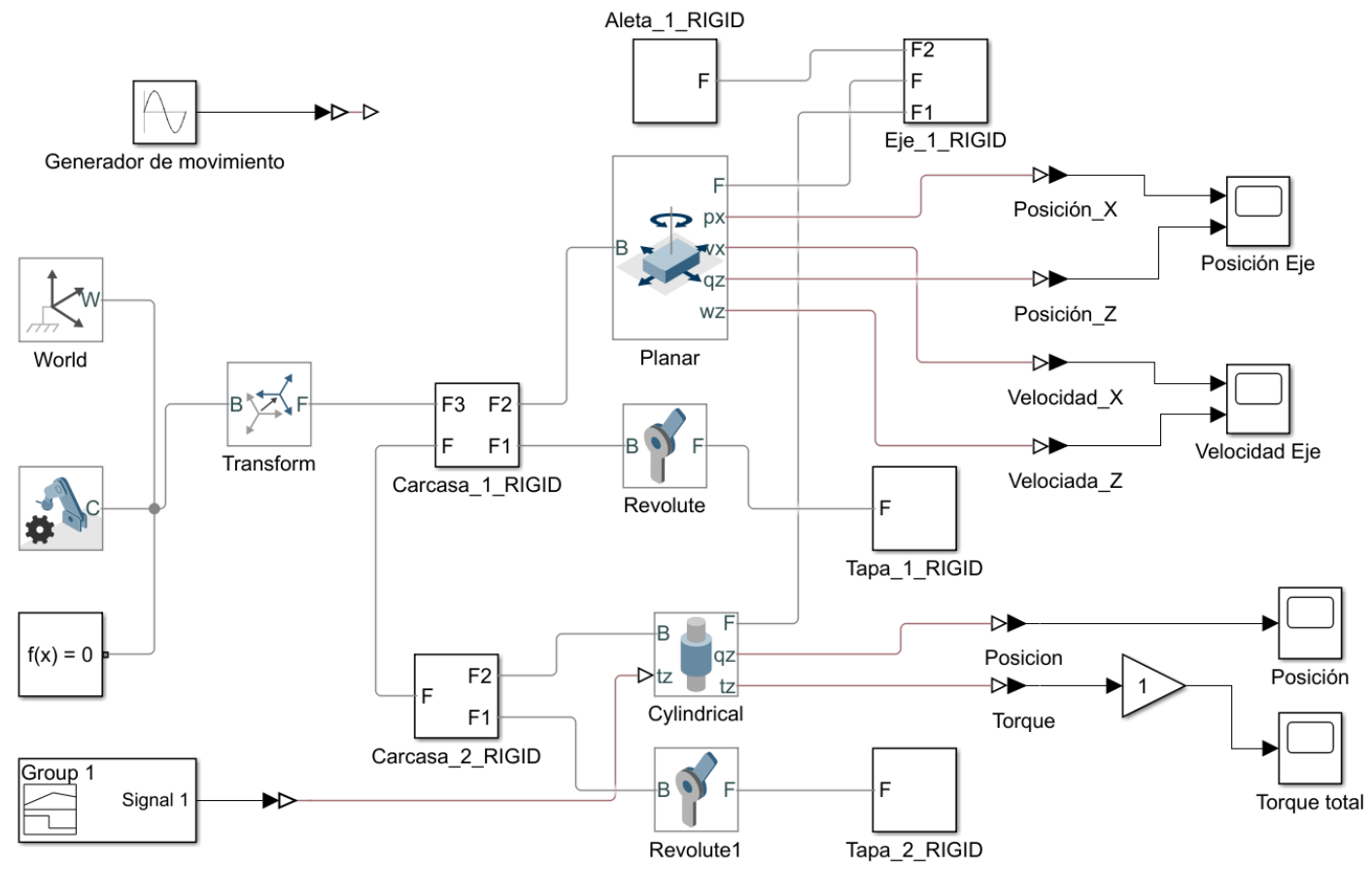

Fig. 4. Simscape multicuerpo del mecanismo hidráulico, diagrama de bloques.

Fuente: Elaboración propia. 


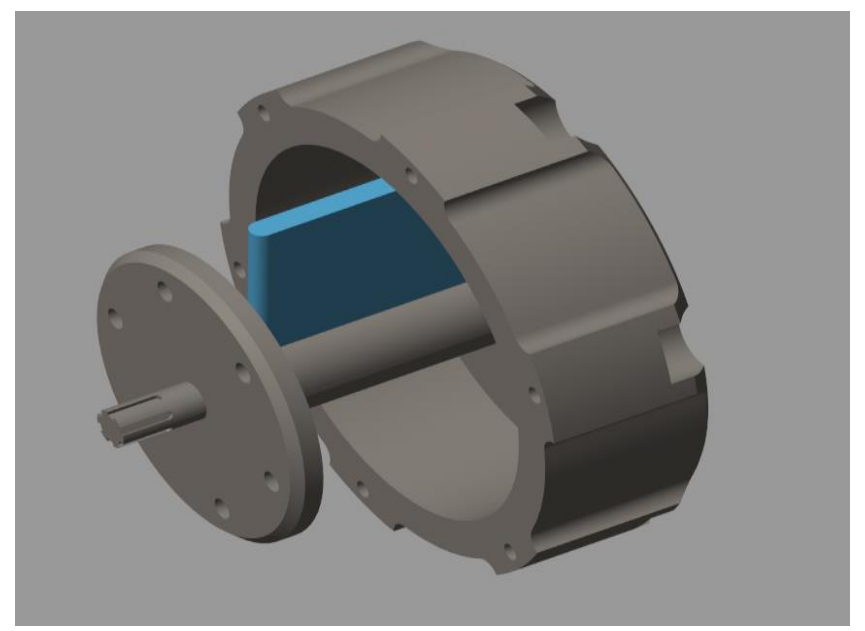

Fig. 5. Mecanismo hidráulico para rehabilitación de rodilla, simulado en simscape.

Fuente: Elaboración propia.

\section{Resultados.}

Según estudios realizados de fuerzas y torques en articulaciones, determinan que un desplazamiento angular de $65^{\circ}$ a $90^{\circ}$ con un par de $4.6 \mathrm{Nm}$ y como resultados se tiene:

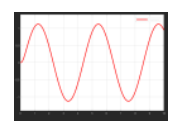

Fig. 6. Posición con movimiento oscilatoria controlado a $1.15 \mathrm{rad}$.

Fuente: Elaboración propia. 


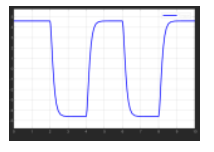

Fig. 7. Par aplicado al mecanismo de $4.6 \mathrm{Nm}$.

Fuente: Elaboración propia.

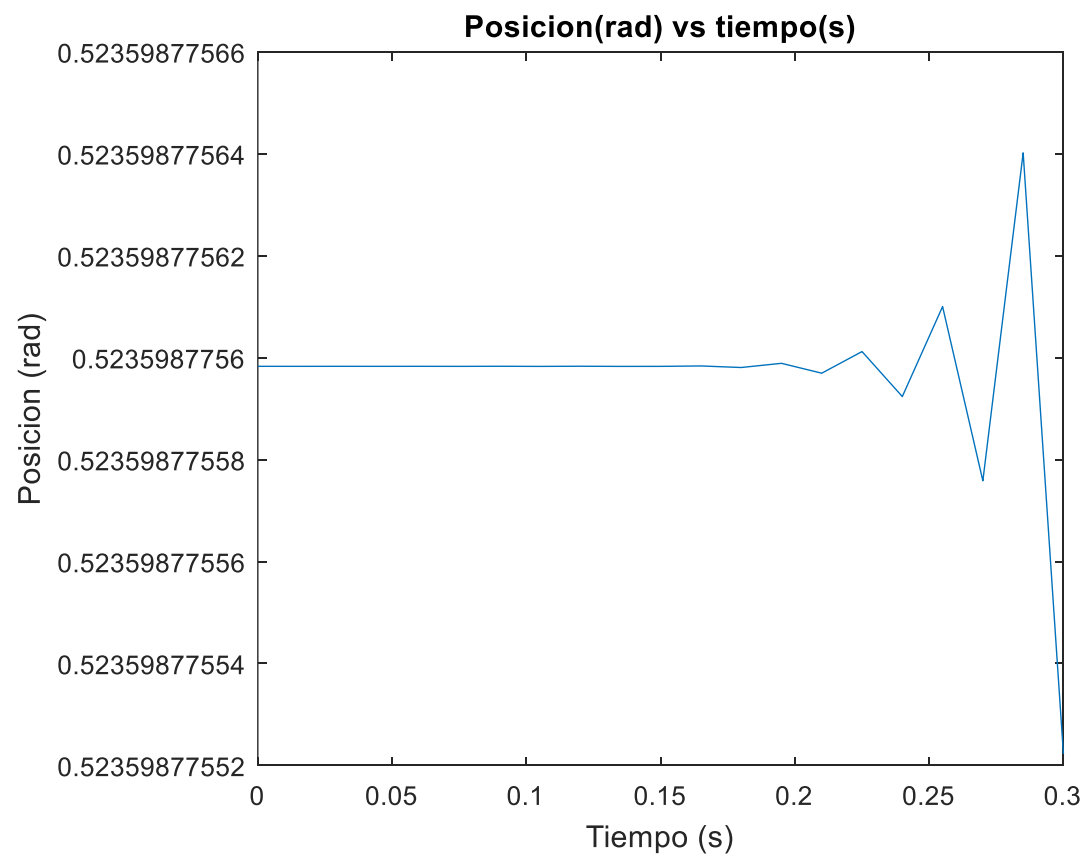

Fig. 8. Posición vs tiempo.

Fuente: Elaboración propia. 


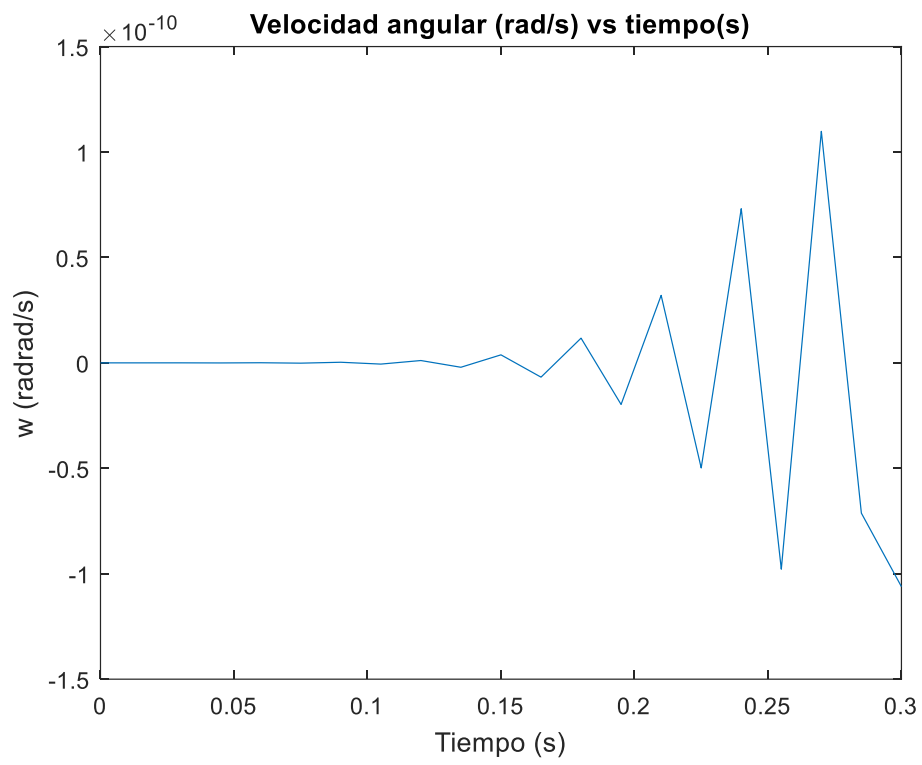

Fig. 9. Velocidad vs tiempo.

Fuente: Elaboración propia.

\section{Conclusiones.}

- Con la graficación del servoactuador se obtiene el modelo matemático que incluye las variables de velocidades de ingreso del difluido y de las dimensiones geométricas de las aletas y de la cámara del actuador.

- La ecuación matemática obtenida no contempla la acción de la fricción y las filtraciones del fluido, lo que puede producir un cambio sustancial al modelo matemático.

- Al realizar la simulación de multicuerpos utilizando Simscape, se puede analizar la posición, velocidad y torque y contrastar con valores existentes y realizar modificación en los modelos existentes, y como último paso se podría realizar un control del actuador.

\section{Referencias bibliográficas.}

[1] M. A. Naya, “Aplicación de la dinámica multicuerpo en tiempo real a la simulación y el control de automóviles," 2007.

[2] A. A. Shabana, Dynamics of multibody systems. Cambridge: Cambridge University Press, 2013.

[3] K. Russell, Kinematics and Dynamics of Mechanical Systems Implementation in MATLAB® and Simmechanics ${ }^{\circledR}$. Boca Raton : CRC Press, Taylor \& Francis, 2018.: CRC Press, 2018. 
[4] P. J. Kumar, E. J. McPherson, L. D. Dorr, Z. Wan, and K. Baldwin, "Rehabilitation after total knee arthroplasty: a comparison of 2 rehabilitation techniques," Clin. Orthop. Relat. Res., vol. 331, pp. 93-101, 1996.

[5] M. I. V. Orselli and M. Duarte, "Joint forces and torques when walking in shallow water,” J. Biomech., vol. 44, no. 6, pp. 1170-1175, 2011. 


\section{PARA CITAR EL ARTÍCULO INDEXADO.}

Vera Luzuriaga, J., Buñay Guaman, J. S., \& Pozo Safla, E. R. (2019). Simulación de un actuador hidráulico sumergible para rehabilitación de rodilla. Ciencia Digital, 3(4.2), 228-240.

https://doi.org/10.33262/cienciadigital.v3i4.2.1091

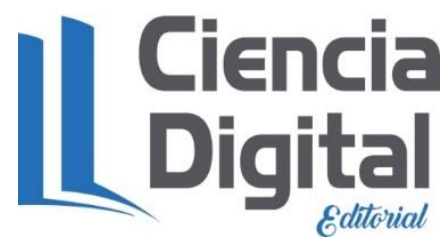

El artículo que se publica es de exclusiva responsabilidad de los autores y no necesariamente reflejan el pensamiento de la Revista Ciencia Digital.

El artículo queda en propiedad de la revista y, por tanto, su publicación parcial y/o total en otro medio tiene que ser autorizado por el director de la Revista Ciencia Digital.
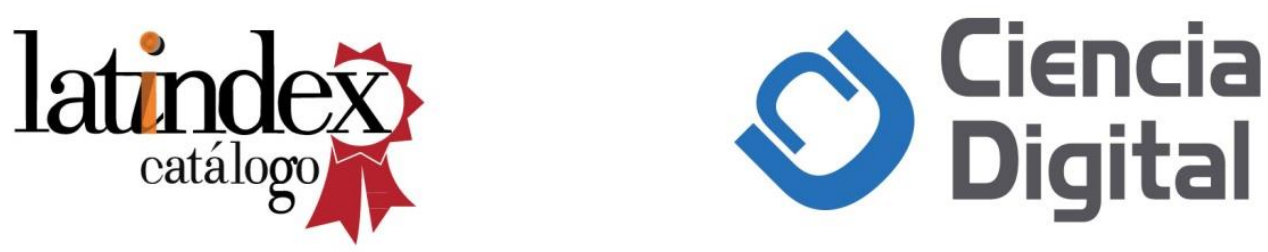